\title{
Risk factors for breast cancer: relevance to screening
}

\author{
S W DUFFY, ${ }^{1}$ M MAUREEN ROBERTS, ${ }^{2}$ AND R A ELTON ${ }^{1}$ \\ From the Medical Computing and Statistics Unit, ${ }^{1}$ University of Edinburgh, and the Edinburgh Breast \\ Screening Clinic, ${ }^{2}$ Edinburgh EH11 2JE, UK
}

SUMMARY In a study of risk factors for breast cancer in women aged between 40 and 60 information was obtained from 236 cases identified at a diagnostic clinic and 2962 controls with no breast abnormality who attended a screening clinic. Statistical analysis of the data used information on a further 167 women who had attended both clinics to correct for possible between clinic bias in questionnaire responses. Relative risk was estimated in four 5 year age groups, and a significant excess risk was found for women under 55 who had undergone previous breast biopsy and for women aged 50-55 who were premenopausal or who had a family history of breast cancer. No significant risk was found to be associated with marital status, age at first pregnancy, breast feeding, or use of hormonal drugs. It is concluded that epidemiological information is of little value in identifying high risk groups for a screening programme in this age range.

In the past three or four decades many studies have endeavoured to determine aetiological factors in breast cancer and investigate their effect on relative risk of the disease. ${ }^{1}$ The most commonly observed factors have been race, age, menstrual history, parity, age at first birth, family history of breast cancer, and a history of benign disease of the breast. Other factors of possible importance are socioeconomic groups, diet and body weight, previous radiation exposure, previous use of hormonal drugs, and history of early abortion.

The study we report here differs from the normal case control design in that the retrospective information used to compare cases and controls was collected from two different populations of women. Although there are obvious disadvantages in terms of possible bias between groups when they are studied independently, our study has a major advantage in that a large random sample of the normal population was available as a control group, drawn from the same demographic area as the cases.

The two groups of women studied were

(1) women referred to a surgical diagnostic breast clinic because of symptoms of breast disease; and

(2) well women from general practitioner lists invited to attend a screening clinic.

For both groups, epidemiological information was recorded on precoded forms and the data were computerised. Our cases of breast cancer were consecutive series of patients from the diagnostic clinic, and the controls were women found to be normal at the screening clinic. The risk factors recorded were marital status, menstrual status, age at menopause, reproductive history, history of breast feeding, use of oral contraceptives and non-contraceptive oestrogenic hormones, history of breast disease, and family history of breast cancer. The statistical analysis of risk factors included adjustment for any bias between clinics, based on the data from a sample of women who had attended both.

\section{Patients}

Information was available on 3328 women aged from 9 to 90 attending the diagnostic clinic of the department of clinical surgery, Royal Infirmary of Edinburgh, from 1974 to 1978 , of whom 504 were finally diagnosed as having breast cancer, 1912 as having other benign disorders of the breast, and 912 as having no serious abnormality. Of the women attending the Edinburgh Breast Screening Clinic, information was available on 3952 asymptomatic women aged between 40 and 60 who attended between 1975 and $1978 .{ }^{2}$ Of this group, only 10 were diagnosed as having breast cancer at their first visit. Of the rest, 76 had lumps that biopsy showed to be benign and a further 876 on clinical or mammographic examination had diffuse benign changes. The rest were deemed normal. For our current study only women aged 40-59 were included, yielding 236 cases of breast cancer from the diagnostic clinic and the 2962 normal controls from the screening clinic. 


\section{Comparison of the two sets of data}

As a first step, the two sets of forms were discussed in depth to determine which risk factors could be studied. The two doctors who had designed the forms had been trained in the same unit, and it was concluded that comparable data were available on all risk factors listed in the previous section.

A total of 167 women had attended both clinics within six months, with complete records available on 109 women. As there is established liability to observer variation in this field, ${ }^{3}$ we examined these data to determine the degree of bias between clinics, with the results shown in table 1 . Using a likelihood based statistical technique, revised estimates of relative risk were derived and significance tests for a difference from unity performed. ${ }^{4}$ In the application of this technique the true state was assumed to be that reported in the screening clinic, so the revised estimates may be regarded as the relative risks associated with reporting of the risk factors in the screening clinic. Variation arose generally from consistently negative reporting in the diagnostic clinic, suggesting that the screening clinic's procedures of recording information were more thorough. For example, of those for whom a positive history of breast feeding was recorded in the screening clinic, $29 \%$ had no such history recorded in the diagnostic clinic, whereas the corresponding figure for a positive history recorded in the diagnostic clinic and a negative in the screening clinic was only $10 \%$.

The relative risk was calculated as follows:

Let $p_{1}$ and $p_{2}$ be the reported rates of occurrence of a given risk factor in the cases and controls respectively. Let $q_{1}$ and $q_{2}$ be the true and false positive rates for the reporting of that factor in the diagnostic clinic, based on the data on women attending both clinics and the assumption that the reporting of the factor in the screening clinic is correct. Then the relative risk is estimated as

$$
\begin{aligned}
& r_{1}\left(1-r_{2}\right) / r_{2}\left(1-r_{1}\right), \text { where } \\
& r_{1}=\left(p_{1}-q_{2}\right) /\left(q_{1}-q_{2}\right) \text { and } \\
& r_{2}=p_{2} .
\end{aligned}
$$

Thus a direct comparison was made between cases with cancer and their controls within each age group.

It should be noted that with this method relative risks are not estimable when the proportion of cases reporting risk factor positive exceeds the proportion of true positives in the data on women attending both clinics and when the proportion of false positives in the data on women attending both clinics exceeds either the proportion of cases reporting risk factor positive or the proportion of true positives.

The first two of these cases correspond to all the cases of breast cancer reporting the risk factor positive (a relative risk of "infinity") and to all the cases of breast cancer reporting the risk factor negative (a relative risk of zero).

\section{Results}

Within the age range used for the study the age distributions of cases and controls were similar (table 2). For the analysis of risk factors, the women were divided into four age groups $(40-44,45-49,50-54$, and 55-59), so that the effects of potential risk factors could be investigated separately in each age group.

The frequency distributions of the risk factors studied and their calculated relative risk estimates

Table 1 Screening clinic and diagnostic clinic responses for women who attended both clinics within six months and for whom the information was recorded

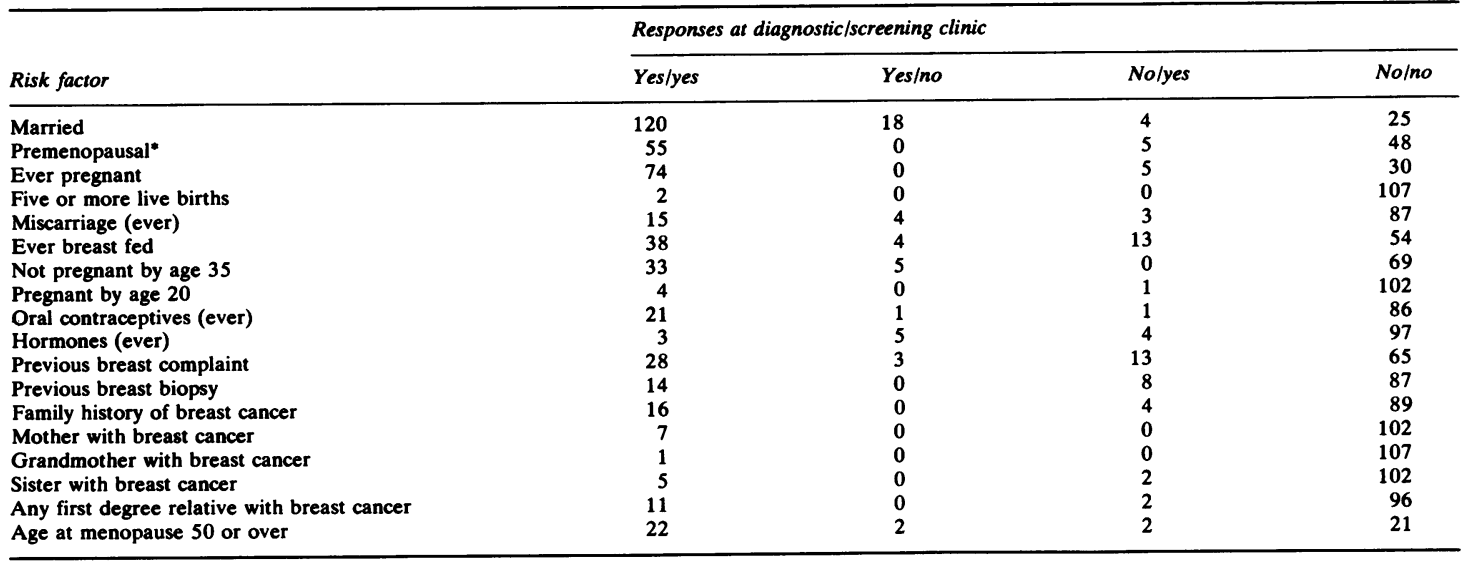

*Premenopausal defined as having had a period within six months. A few women who had undergone hysterectomy were included in the postmenopausal group. 
Table 2 Observed age distribution of cases and controls

\begin{tabular}{lrl}
\hline Age range & $\begin{array}{l}\text { No of cases } \\
(\%)\end{array}$ & $\begin{array}{l}\text { No of controls } \\
(\%)\end{array}$ \\
\hline $40-44$ & $49(20 \cdot 8)$ & $627(21 \cdot 2)$ \\
$45-49$ & $60(25 \cdot 4)$ & $752(25 \cdot 4)$ \\
$50-54$ & $72(30 \cdot 5)$ & $834(28 \cdot 1)$ \\
$55-59$ & $55(23 \cdot 3)$ & $749(25 \cdot 3)$ \\
All ages & $236(100 \cdot 0)$ & $2962(100 \cdot 0)$ \\
\hline
\end{tabular}

adjusted for between-clinic bias are given in table 3 .

A highly significant increase in risk $(p<0.005)$ was associated with premenopausal state in women aged 50-54. In women aged 40-44 a significant excess risk was associated with having five or more live births $(p<0 \cdot 05)$. For women with a history of benign breast disease, significant increases in risk were observed, particularly for those who had undergone biopsy and for those in the younger age groups. The effect of previous complaint lost its statistical significance when women with a previous biopsy were excluded, indicating that a history of breast biopsy may be the real influence on risk. A significant increase in risk was also observed in women aged 50-54 who had a first degree relative who had had breast cancer. No significant differences in risk were found for the other factors including history of lactation, oral contraceptives, or hormones.

The analysis of the effects of the factors relating to pregnancy and lactation included 644 non-parous women. Table 4 shows a reanalysis of the effects of the number of live births, history of miscarriage, age at first pregnancy, and breast feeding excluding the non-parous women. The results for the effects of breast feeding, history of miscarriage, and a total parity of five or more were much the same as those for which the non-parous women were included, except that the effect of five or more live births among women aged 40-44 was no longer significant. For age at first pregnancy of 35 or more, some changes were noted, none of them significant.

Because women who were still premenopausal at age 50-54 seemed to be at increased risk (table 3), we estimated the relative risk for age at menopause 50 or over for postmenopausal women of all ages. When analysed in this way, no significant effect was observed (relative risk estimate of 1.20). The mean age at menopause was 46.77 for cases and 46.38 for controls, but it must be remembered that only about half the total group have so far reached the menopause.

\section{Discussion}

By using the methods described, we have found that in our population of women aged 40-60 the risk factor of greatest importance was a history of breast

Table 4 Relative risk estimates for birth and breast feeding factors excluding non-parous women

\begin{tabular}{lllll}
\hline & \multicolumn{4}{l}{ Relative risk estimate for age groups } \\
\cline { 2 - 5 } Risk factor & $40-44$ & $45-49$ & $50-54$ & $55-59$ \\
\hline Five or more live births & 2.97 & 1.25 & 0.68 & 1.42 \\
Miscarriage (ever) & 0.59 & 1.12 & 0.81 & 0.66 \\
Ever breast fed & 0.82 & 0.52 & 1.39 & 0.65 \\
Not pregnant by age 35 & 1.26 & 0.74 & 0.89 & 0.56 \\
Pregnant by age 20 & 0.91 & 1.36 & 1.16 & 1.72 \\
\hline
\end{tabular}

Table 3 Numbers of cases and controls with risk factors present, and age specific relative risk estimates adjusted for between clinic bias. Because of missing information, not all percentages are based on the same denominators

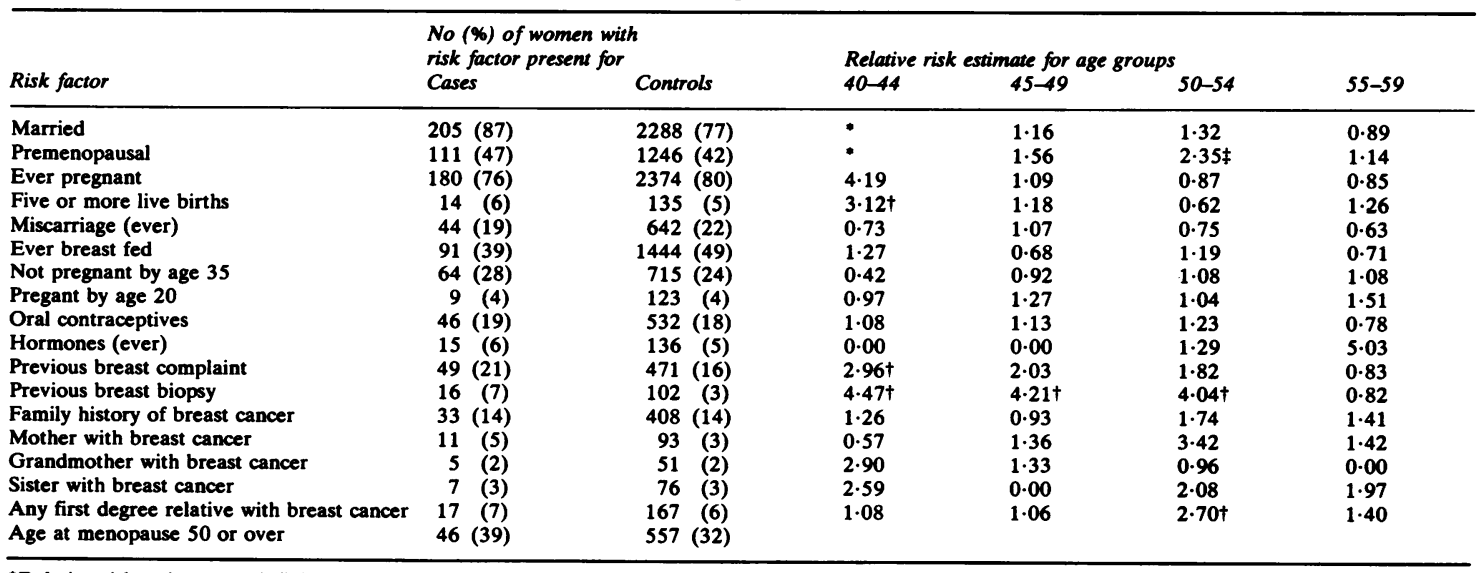

"Relative risk estimate $=$ "infinity." 
biopsy. A family history of breast cancer or a late menopause were associated with increased risk only in certain age groups. We were unable to confirm that nulliparity or late age (35 or over) at first pregnancy were significant risk factors.

We realise that the methods used in our study are not those of the normal case control comparison. Nevertheless, our large control group (12 controls for every case) consisted of women who were not only asymptomatic but had been proved normal by screening and who were invited well women from the lists of general practitioners. The response rate to the invitation was $82 \%$, so we conclude that these women were as representative of the normal population as one could achieve. By contrast, most conventionally designed case control studies use hospital based controls who are not necessarily representative of the population, or a community based sample collected specifically for this purpose and therefore often not sampled with the same degree of thoroughness or validity as were our controls. The two reports currently available of risk factors in screening programmes have certain disadvantages. One, from the New York Health Insurance Plan study, had only a $60 \%$ response to invitation and the other from the current American Breast Cancer Detection Programme, included self-selected women..$^{56}$

The main disadvantage of our study was the merging of information that had been collected independently at two clinics. We believe that we have overcome this problem by making use of the data on a sample of women who attended both clinics and who therefore gave direct information on the extent and direction of any biases between clinics in the response given to the various questions. This information has been incorporated in the statistical analysis, for which the method is described elsewhere. ${ }^{4}$ We may therefore have a more trustworthy comparison between cases and controls than is obtained from more conventional studies, in which an absence of recall bias is assumed and no observer variation studies are done. ${ }^{7}$ Assuming, therefore, that the results of our final analyses are reliable, it is pertinent to compare our findings with those of other studies, especially those based on community controls.

\section{History of benign diseases}

Most other groups have found an increase in risk with a history of breast biopsy, some suggesting that the increase occurs only when atypical epithelial cells are present. ${ }^{89}$ Unfortunately, we were not able to study pathological aspects of the disease. A report of a separate investigation into the effects of previous benign disease of the breast in women attending the diagnostic and screening clinics found no association between this variable and a final diagnosis of breast cancer. ${ }^{10}$ This may be interpreted as indicating that previous breast biopsy does not help in predicting the current diagnosis, even though it may be a risk factor.

\section{Family history}

One of our more surprising findings is that a family history of breast cancer was found to increase risk in only one age group. A possible explanation for ourô negative findings might be selection bias in our control group (women might be more likely to attendō. for screening if they have a family history of breast $+\omega$ cancer). The incidence in our control group, however, is less than in most reported studies, even those using community controls, and considerable variation is found (table 5). The incidence of a family history in our cases is also lower than in many reported series. Possible explanations for this include statistical chance, our age range, or genuine differences between Scottish women and others in the epidemiology of their disease, but we are unable to clarify this question without further study.

Table 5 Percentage of cases and controls with family history of breast cancer and incidence of nulliparity in the present study and others

\begin{tabular}{|c|c|c|c|c|c|c|c|c|c|}
\hline \multirow[b]{3}{*}{ References } & \multirow[b]{3}{*}{ Type of controls } & \multicolumn{4}{|c|}{ Family history } & & & & \\
\hline & & \multicolumn{2}{|c|}{ Mother } & \multicolumn{2}{|l|}{ Sister } & \multicolumn{2}{|c|}{ Nulliparity } & \multicolumn{2}{|c|}{ No studied } \\
\hline & & $\begin{array}{l}\text { Cases } \\
\text { (\%) }\end{array}$ & $\begin{array}{l}\text { Controls } \\
(\%)\end{array}$ & $\begin{array}{l}\text { Cases } \\
(\%)\end{array}$ & $\begin{array}{l}\text { Controls } \\
(\%)\end{array}$ & $\begin{array}{l}\text { Cases } \\
(\%)\end{array}$ & $\begin{array}{l}\text { Controls } \\
1 \%\end{array}$ & $\begin{array}{l}\text { Cases } \\
(\%)\end{array}$ & $\begin{array}{l}\text { Controls } \\
(\%)\end{array}$ \\
\hline Present & Screening $(40-60)$ & $4 \cdot 7$ & $3 \cdot 2$ & $3 \cdot 0$ & 3.0 & 24 & 20 & 236 & 2962 \\
\hline HIP" & Screening (40-64) & $18 \cdot 0$ & $18 \cdot 0$ & $12 \cdot 0$ & $7 \cdot 0$ & 24 & 18 & 101 & 9914 \\
\hline BCDDP & Screening $(\geqslant 35)$ & $8 \cdot 0$ & $2 \cdot 2$ & - & - & 18 & 17 & 405 & 1156 \\
\hline Bain et al ${ }^{11}$ & Nurses $(30-55)$ & 9.7 & $5 \cdot 6$ & $8 \cdot 0$ & 3.4 & - & - & 1159 & 121964 \\
\hline Adami et al ${ }^{12}$ & Random population & - & - & - & - & 26 & 22 & 179 & 179 \\
\hline Miller et al ${ }^{15}$ & Census (all ages) & - & - & - & - & 25 & +14 & 1600 & 11127 \\
\hline Choi et al ${ }^{13}$ & Neighbourhood (35-74) & - & - & - & - & $12^{*}$ & $11 *$ & 400 & 400 \\
\hline Pfaffenberger et $a^{14}$ & Hospital & 8.6 & $4 \cdot 4$ & $7 \cdot 3$ & 5.5 & 23 & 17 & 1415 & 2516 \\
\hline
\end{tabular}

* Only married women included.

HIP = Health Insurance Plan.

BCDD̄ $\bar{P}=$ Breast Cancer Detection Demonstration Programme. 


\section{Reproductive factors}

Continued menstruation after the age of 50 increased the risk for women aged 50-54 in our series, but the effect of age at menopause was not significant when only postmenopausal women were considered. Most studies are in agreement that a late menopause is a risk factor, but the major effect is seen in women over the age of $70 . .^{16}$ As our population is aged 60 or under, we are unlikely to see the full effect of late menopause in our study.

In our population neither nulliparous women nor those who had first pregnancies after the age of 35 appeared to be at increased risk, in contrast with other studies. As table 5 shows, the incidence of nulliparity in the groups of women studied varied, but some groups included only married women. The key study that indicated increased risk for late first pregnancy ${ }^{17}$ used hospital controls, but there is divergence of opinion among more recent reports about this factor, and disagreement on whether it affects premenopausal or postmenopausal women. The socioeconomic status of the controls (believed to be generally lower in hospital than in community controls) may also be important.

Our findings of an increased risk associated with more than five pregnancies in younger women may be anomalous, but it is of interest that Miller et $a^{15}$ suggested a possible increase of risk in women under 35 who had had more than four pregnancies. The reason for this is obscure.

\section{Conclusion}

From our studies of women aged 40-60 in Edinburgh, we conclude that a small proportion of women in the normal population are at increased risk if they have a history of breast biopsy, and possibly if they have a late menopause or a close family relative with breast cancer, particularly in certain age groups. Such women may have a relative risk of about twice that for the rest of the population. Nevertheless, since only a small proportion of the population gave a history of such factors (3.4\% for previous biopsy, $5.7 \%$ for a first degree relative with the disease), it seems unlikely that screening offered only to women at high risk in this age group would be of clinical relevance. Care should be exercised in telling women that they are at higher risk than normal, as this may be unjustifiable and create high levels of anxiety. It is not impossible that stress itself may be a risk factor for breast cancer.
This work was supported by a project grant from the Medical Research Council. We are grateful to Professor A P M Forrest and the Edinburgh Breast Screening Project Committee for access to records and to Mrs R Wood and Mrs F Stent for help with the computerisation of the data.

Correspondence should be addressed to $\operatorname{Dr} M \mathrm{M}$ Roberts.

\section{References}

${ }^{1}$ Kalache A. Risk factors for breast cancer: a tabular summary of the epidemiological literature. Br J Surg 1981; 68: 797-9.

${ }^{2}$ Edinburgh breast screening clinic. Screening for breast cancer. Br Med J 1978; ii: 175-8.

${ }^{3}$ Yorkshire breast cancer group. Observer variation in recording clinical data from women presenting with breast lesions. $\mathrm{Br}$ Med J 1977; ii: 1196-9.

4Elston RA, Duffy SW. Correcting for the effect of misclassification bias in a case-control study using data from two different clinics. Biometrics (in press).

${ }^{5}$ Shapiro S, Strax P, Venet L, Fink R. The search for risk factors in breast cancer. Am J Public Health 1968; 58: 820-35.

${ }^{6}$ Brinton LA, Williams RR, Hoover RN, Stegens NL, Feinleib L, Fraumeni JF. Breast cancer risk factors among screening programme participants. J Natl Cancer Inst 1979; 62: 37-44.

${ }^{7}$ Sackett DL. Bias in analytical research. $J$ Chronic Dis 1979; 32: 51-63.

${ }^{8}$ Hutchinson WB, Thomas DB, Hamlin WB, Roth GJ, Peterson AV, Williams B. Risk of breast cancer in women with benign breast disease. J Natl Cancer Inst 1980; 65: 13-20.

9 Page DL, Vander Zwaag R, Rogers LW, Williams LT, Walker EW, Hartmann WH. Relation between component parts of fibrocystic disease complex and breast cancer. $J$ Natl Cancer Inst 1978; 61: 1055-63.

${ }^{10}$ Chetty U, Wang CC, Forrest APM, Roberts MM. Benign breast disease and cancer. Br J Surg 1980; 67: 789-90.

${ }^{11}$ Bain C, Speizer FE, Rosner B, Belanger C, Hennekens $\mathrm{CH}$. Family history of breast cancer as a risk indicator for the disease. Am J Epidemiol 1980; 111: 301-8.

${ }^{12}$ Adami HO, Rimsten A, Stenkvist B, Vegelius J. Reproductive history and risk of breast cancer. Cancer 1978; 41: 747-57.

${ }^{13}$ Choi NW, Howe GR, Miller AB, et al. An epidemiologic study of breast cancer. Am J Epidemiol 1978; 107: 510-21.

14 Paffenbarger RS, Kampert JB, Chang HG. Characteristics that predict risk of breast cancer before and after the menopause. Am J Epidemiol 1980; 112: 258-68.

${ }^{15}$ Miller AB, Barclay THC, Choi NW, et al. A study of cancer, parity and age at first pregnancy. J Chronic Dis 1978; 33: 595-605.

${ }^{16}$ Trichopoulos D, MacMahon B, Cole P. Menopause and breast cancer risk. J Natl Cancer Inst 1972; 48: 605-13.

${ }^{17}$ MacMahon B, Cole P, Lin TM, et al. Age at first birth and breast cancer risk.Bull WHO 1970; 43: 209-21. 